\title{
Spin-orbit coupling effect on electronic, linear and nonlinear optical properties of Bi2S3 and the Ternary Bismuth Sulfide Bi2S2.75Se0.25: Ab-initio calculations
}

Houda Ben Abdallah ( $\nabla$ bhouda02@yahoo.fr)

University of Tunis El Manar: Universite de Tunis El Manar https://orcid.org/0000-0003-4006-9654

Walid Ouerghui

Université de Carthage: Universite de Carthage

\section{Research Article}

Keywords: Electronic structure, Optical properties, Spin-orbit coupling, Bismuth sulfide

Posted Date: July 6th, 2021

DOl: https://doi.org/10.21203/rs.3.rs-427791/v1

License: (c) (1) This work is licensed under a Creative Commons Attribution 4.0 International License. Read Full License

Version of Record: A version of this preprint was published at Optical and Quantum Electronics on November 29th, 2021. See the published version at https://doi.org/10.1007/s11082-021-03411-y. 
Spin-orbit coupling effect on electronic, linear and nonlinear optical properties of $\mathrm{Bi}_{2} \mathrm{~S}_{3}$ and the Ternary Bismuth Sulfide $\mathrm{Bi}_{2} \mathrm{~S}_{2.75} \mathrm{Se}_{0.25}$ : Ab-initio calculations

\author{
H. Ben Abdallah ${ }^{\mathrm{a}, *}$, W. Ouerghui ${ }^{\mathrm{b}}$ \\ ${ }^{a}$ Laboratoire de Physique de La Matiere Condensée, Département de Physique, Faculté Des \\ Sciences de Tunis, Université Tunis El Manar, 2092, Tunis, Tunisia \\ ${ }^{\mathrm{b}}$ Laboratoire Matériaux, Molécules et Applications, Institut Préparatoire aux Etudes Scientifiques \\ et Techniques. University Carthage. La Marsa 2075, Tunis, Tunisia. \\ * Correspondence author \\ E-mail adress: bhouda02@yahoo.fr (H. Ben Abdallah)
}

\begin{abstract}
Although the relevant properties of the bismuthinite $B i_{2} S_{3}$, it was recently approved that the substitution of Se atoms in the $B i_{2} S_{3}$ lattice can significantly enhance its electro-optical properties. In the present work, a detailed study on the structural, electronic and optical properties of $B i_{2} S_{2.75} S e_{0.25}$ has been carried out based on first principle calculations. The simultaneous effect of Se-doping and spin-orbit coupling (SOC) on bismuth sulfide $B i_{2} S_{3}$ was investigated. Our calculations show that $B i_{2} S_{2.75} S e_{0.25}$ exhibits a narrow direct band gap of $1.062 \mathrm{eV}$ after inclusion of the (SOC). The calculation of the carrier effective masses indicates that $B i_{2} S_{2.75} S e_{0.25}$ may possess a high electron mobility material which is in accordance with experimental studies. The linear absorption optical spectra for both $\mathrm{Bi}_{2} S_{3}$ and $B i_{2} S_{2.75} S_{0.25}$ show that doping bismuthinite with (Se) increases the optical absorption coefficient in the visible range and takes a value up to $1010^{5} \mathrm{~cm}^{-1}$. In addition, the dielectric function, optical conductivity and the energy loss function of $B i_{2} S_{3}$ and $B i_{2} S_{2.75} S e_{0.25}$ were also derived. The addition of the (Se) content induces a red shift in agreement with experimental studies. A noticeable effect of the (SOC) on the linear optical parameters was observed. The stability of the excitons was also studied by the estimation of the binding energy value. The dispersion energy parameters of $B i_{2} S_{3}$ and $B i_{2} S_{2.75} S e_{0.25}$ were estimated using a single oscillator model. Some nonlinearities have been computed with and without inclusion of (SOC) showing that $B i_{2} S_{2.75} S e_{0.25}$ with large nonlinear optical parameters is promising candidate in photonic switching applications.
\end{abstract}

Keywords: Electronic structure, Optical properties, Spin-orbit coupling, Bismuth sulfide 


\section{Introduction}

Bismuth chalcogenide materials $\mathrm{Bi}_{2} \mathrm{X}_{3}(\mathrm{X}=\mathrm{S}, \mathrm{Se}, \mathrm{Te})$ are considered as the second generation topological insulators as they have insulating bulk band gap and conducting states on their surface [1]. Their bulk band gaps are generated because of the inherent spin-orbit coupling in these systems. The cation $\mathrm{Bi}^{3+}$ has a large relativistic effect and a strong sp hybridization in the valence band [2]. As a result, the Bi based materials may possess low carrier effective masses and therefore high carrier mobility leading them to be very useful in photovoltaic applications as a solar cell. Particularly, VI- bismuth materials $\mathrm{Bi}_{2} \mathrm{X}_{3}$ are functional compounds [3] and very attractive materials for several applications including: photovoltaics [4], thermoelectrics [5], photoelectrochemicals [6], photocatalytic activity [7] , micro- and optoelctronic devices [8] and infrared detectors [9]. Among $\mathrm{Bi}_{2} \mathrm{X}_{3}$ materials, the bismuth sulfide $B i_{2} S_{3}$-bismuthinite- is a metal chalcogenide with a very attractive and intriguing properties : a large optical absorption coefficient $\left(>10^{5} \mathrm{~cm}^{-1}\right.$ for $\lambda<500 \mathrm{~nm}$ ), high electron mobility $\left(>500 \mathrm{~cm} \mathrm{~V}^{-1} \mathrm{~s}^{-1}\right)$ [10] and so on. The band gap of $\mathrm{Bi}_{2} \mathrm{~S}_{3}$ is around 1.3-1.7 eV [11] and therefore lies in the visible light spectrum allowing it to be a light-absorber material in solar cells [12]. These relevant properties have allowed $B i_{2} S_{3}$ to be a potential candidate for diverse domains such as photovoltaics [13], thermoelectrics [14], electrochemical hydrogen storage [15], lithium and sodium batteries [16], electrochemical sensors [17], X-ray computed tomography [18], and so on. Despite its interesting and relevant properties, the use of $\mathrm{Bi}_{2} \mathrm{~S}_{3}$ in the various fields mentioned above is hampered by its large electrical resistivity [19] if it is used as the electron acceptor and its short lifetime of minority carriers if it is used in solar cells. To improve the efficiency of $\mathrm{Bi}_{2} \mathrm{~S}_{3}$ in solar cells development and to enhance its electrical transport properties, a systematic study on intrinsic and extrinsic defects is crucial to optimize performances of bismuth sulfide $B i_{2} S_{3}$. Several researches have carried out a comprehensive study on intrinsic defects and doping in $B i_{2} S_{3}$. For example, H. Mizoguchi et al. [20] found that $\mathrm{S}$ vacancies in the lattice of bismuthinite can reduced its electrical resistivity by 23 orders of magnitude. Similarly, doping $\mathrm{Bi}_{2} \mathrm{~S}_{3}$ by substitutional extrinsic elements such as $\mathrm{Cl}$ [21], $\mathrm{Sb}$ [22], $\mathrm{Cu}$ [23], $\mathrm{Ag}$ [24], $\mathrm{Eu}$ [25] and $\mathrm{Mn}$ [26] has been found to optimizing its carrier concentration so improving its electrical properties. Recently, much experimental reports have shown that the introducing selenium atoms to $B i_{2} S_{3}$ can be considered as a very efficient way to increase its electrical and optoelectronic properties. It has shown that Se alloying bismuthinite in $\mathrm{S}$ sites $\left(\mathrm{Bi}_{2} \mathrm{~S}_{2.85} \mathrm{Se}_{0.15}\right)$ could increase a figure of merit (ZT) and enhance the thermal conductivity 
upon Se incorporation [19]. Ajara et al. [10] found that $\left(B i_{2} S_{3-x} S e_{x}\right)$ solid solutions possess interesting and attractive electrical properties than $B i_{2} S_{3}$. Recently, experimental investigations conducted by Ye Chen \&al. [27] found that the power factor in the case of Se alloying at S sites in $B i_{2} S_{3}$ is about 100 times larger than that of undoped $B i_{2} S_{3} . B i_{2} S_{3-x} S e_{x}$ can be also an all-optical photonics switching device due to its intersting non-linear optical properties [28]. Photocatalytic activity can be highly increased when doping selenium into $B i_{2} S_{3}$ photocatalyst [29]. It has also been proved that Se-doped $B i_{2} S_{3}$ can be used as anodes for sodium-ion batteries [30]. In contrast, to our knowledge no theoretical study has been reported to date to investigate changes in electroptical properties caused by the substitution of Se atoms into $B i_{2} S_{3}$. For this purpose, our study is focused on predicting the structural, electronic and optical properties of $B i_{2} S_{3-x} S e_{x}(\mathrm{x}=0$ and 0.25). The calculations were carried out using ab initio density functional theory (DFT) employing the Full-Potential Linearized Plane Wave (FP-LAPW) method. In the present paper we also discussed the effect of the spin-orbit coupling (SOC) on the electro-optical properties for $B i_{2} S_{3}$ and the ternary bismuth sulfide $B i_{2} S_{2.75} S e_{0.25}$.

\section{Computational details}

In the present study, the full potential linearized plane wave (FP-LAPW) method [31] as implemented in the Ab-initio simulation Wien2k [32] package based on the density functional theory (DFT) [33], is used to carried out all of the calculations. Perdew-Burke-Ernzerhof (PBEGGA) scheme was used to describe the exchange-correlation interaction [34] in addition of spinorbit coupling (SOC) [35]. In this study, the valence electrons are: $\left[6 s^{2} 6 p^{3}\right],\left[3 s^{2} 3 p^{4}\right]$ and $\left[4 s^{2} 4 p^{4}\right]$ for $\mathrm{Bi}, \mathrm{S}$ and $\mathrm{Se}$ atoms respectively. The potential and charge density inside the atomic spheres are taken by $l_{\max }=10$. The plane wave cut-off is fixed at $\mathrm{R}_{M T} * \mathrm{~K}_{\max }=8$, where $\mathrm{R}_{\mathrm{MT}}$ denotes the smallest muffin-tin radii of all atomic spheres and $\mathrm{K}_{\max }$ is the largest k-vector of the reciprocal lattice. $\mathrm{G}_{\max }$ $=12(\mathrm{Ryd})^{1 / 2}$ is truncated to develop charge density and the plane wave potential. The energy cutoff for separation core and valence electrons is set to -10 Ry. A $500 \mathrm{k}$-point are used in the irreducible Brillouin zone. A denser k-mesh are used for calculation of the optical properties. The calculations are self-consistently converged when the total energy convergence is fixed to be $10^{-4}$ Ry.

\section{Results and discussion}




\subsection{Structural properties}

Bismuthinite $B i_{2} S_{3}$ crystallizes in orthorhombic structure with the space group pbnm (62) [36]. There are four formula units per unit cell giving rise to 20 atoms in the primitive cell. The crystal structure of $\mathrm{Bi}_{2} \mathrm{~S}_{3}$ consists of five non-equivalent atoms: two non-equivalent $\mathrm{Bi}$ sites and three non-equivalent $\mathrm{S}$ sites. Each $\mathrm{Bi}$ atom is linked to seven atoms of $\mathrm{S}$. Three atoms of $\mathrm{S}$ are linked to $\mathrm{Bi}$ atom by short distances and the other four atoms of $\mathrm{S}$ are linked to Bi by long distances. The Bi-S short-bonds formed a strongly bonded ribbons while the Bi-S long-bonds formed a Van der Waals force introducing a weak interaction between the aligned ribbons [37]. To model the $\mathrm{Bi}_{2} S_{3-x} S e_{x}$ structure, a $2 \times 2 \times 1$ supercell was constructed generating eight $\mathrm{Bi}$ atoms and twelve $\mathrm{S}$ atoms in unit cell with formula $\mathrm{Bi}_{8} \mathrm{~S}_{12}$ in which three $\mathrm{S}$ atoms were replaced by three $\mathrm{Se}$ atoms to get $B i_{2} S_{2.75} S e_{0.25}$. The crystal structures of $B i_{2} S_{3}$ and $B i_{2} S_{2.75} S e_{0.25}$ are shown in Fig. 1.
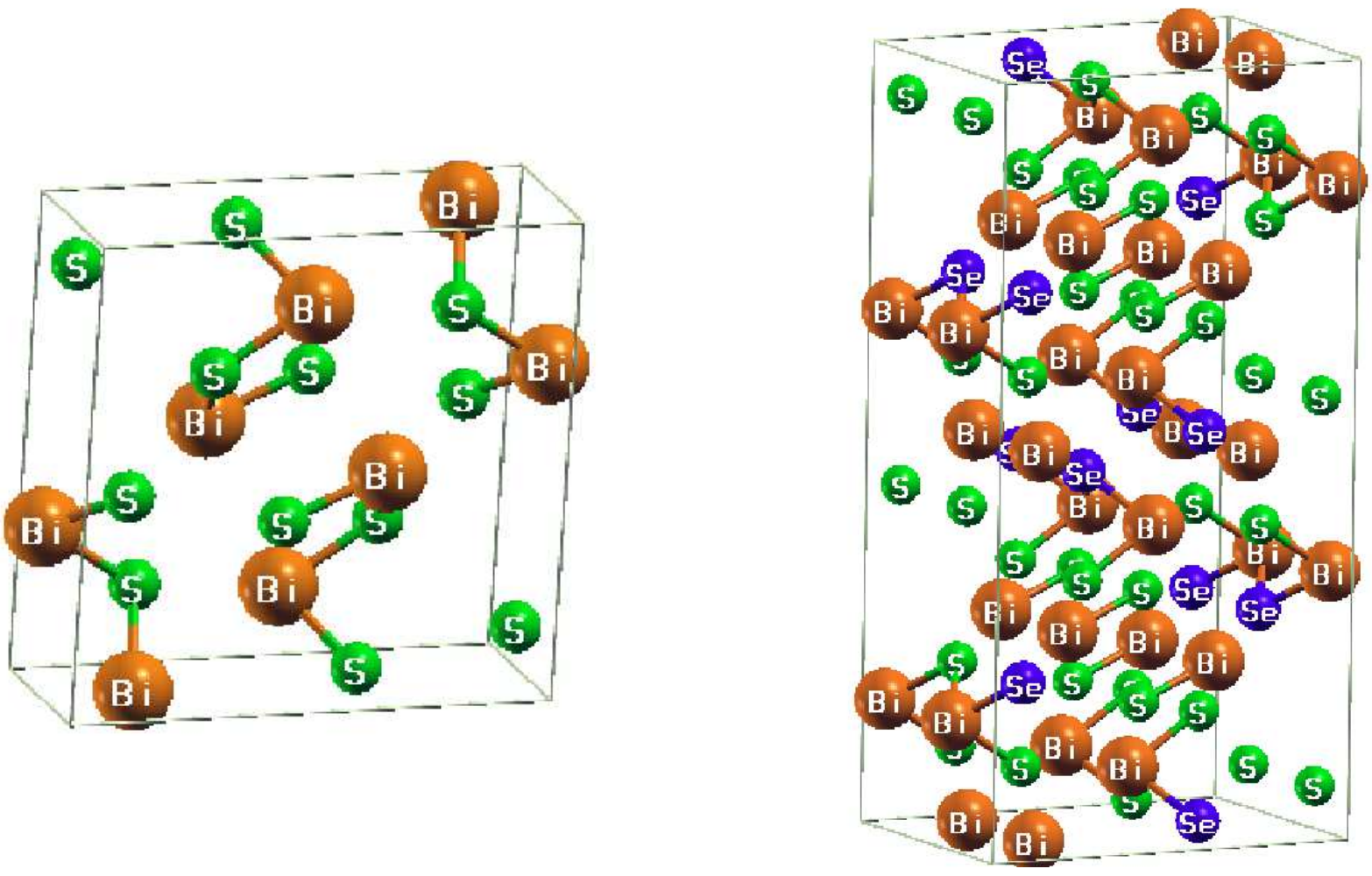

Fig. 1. The orthorhombic crystal structures of $B i_{2} S_{3-x} S e_{x}$. (The left is the structure for $\mathrm{x}=0$ and the right for $\mathrm{x}=0.25$ ). 

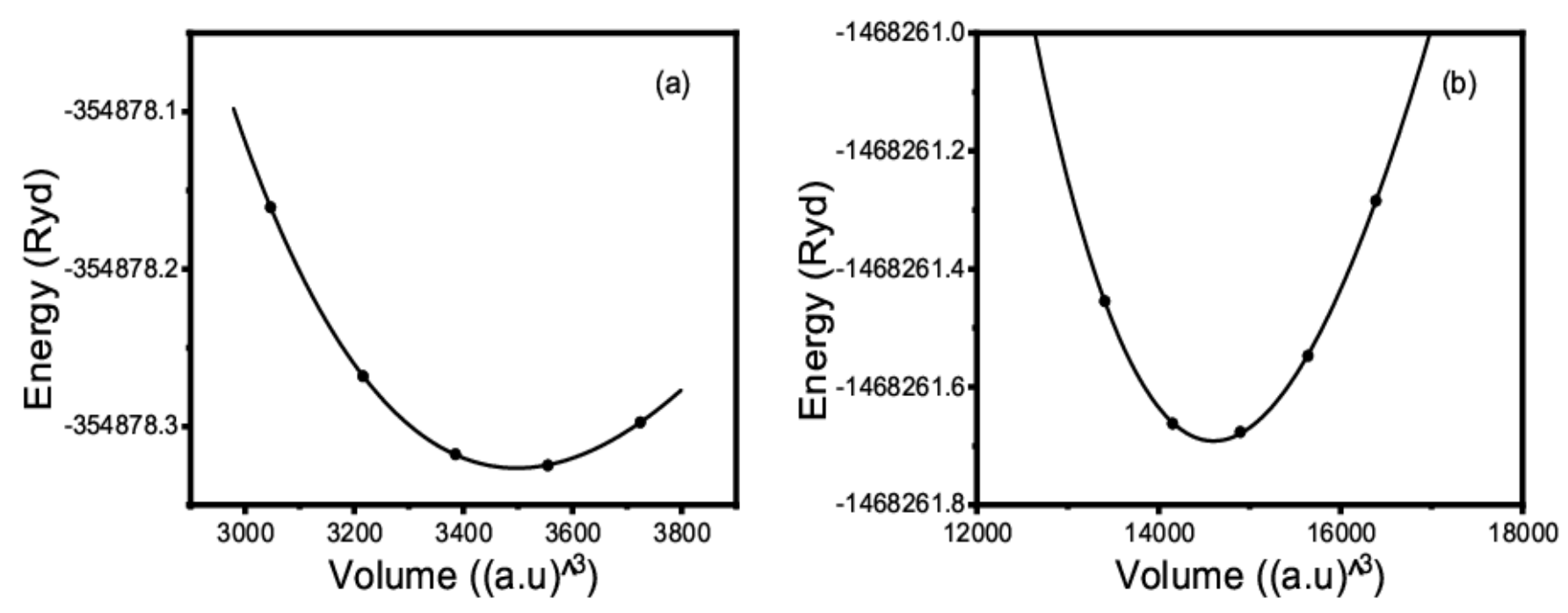

Fig. 2. Calculated total energy as a function of volume within GGA-PBE for $B i_{2} S_{3}$ and $B i_{2} S_{2.75} S e_{0.25}$.

To find the ground state properties of $B i_{2} S_{3}$ and $B i_{2} S_{2.75} S e_{0.25}$, the variation of the total energies as a function of volume is computed within the GGA-PBE method for different volumes about the equilibrium cell volume $\mathrm{V}_{0}$, using the Murnaghan's equation of state [38] as demonstrated in Fig.2. The calculated equilibrium lattice constants, the bulk modulus (B) and its derivative of pressure (B') for both compounds are given in Table1, with other previous theoretical and experimental results. From Table1, we see that the optimized lattice parameters for bismutinite $B i_{2} S_{3}$ are in good agreement with theoretical results and slightly higher than the experimental data since the GGA approximation overestimates the lattice constants. In addition, the lattice constants increase with adding Se in the $B i_{2} S_{3}$ lattice which can be explained by ionic radii difference between $\mathrm{S}^{2-}$ $(1.81 \AA)$ and $\mathrm{Se}^{2-}(1.98 \AA)$. 
Table1 Optimized lattice constants, bulk modulus B and its pressure derived B' and other available experimental data and theoretical values for $B i_{2} S_{3}$ and $B i_{2} S_{2.75} S e_{0.25}$.

\begin{tabular}{|c|c|c|c|c|c|c|}
\hline Compound & Method & $\boldsymbol{a}(\AA)$ & $\boldsymbol{b}(\AA)$ & $\boldsymbol{c}(\AA)$ & $B(G P a)$ & $\boldsymbol{B}^{\prime}$ \\
\hline$B i_{2} S_{3}$ & $\begin{array}{l}\text { Present (GGA-PBE) } \\
\text { Theory (DFT-LDA) [1] } \\
\text { Theory (DFT-GGA) [37 } \\
\text { Experimental [36] }\end{array}$ & $\begin{array}{l}11.428 \\
11.314 \\
11.227 \\
11.305\end{array}$ & $\begin{array}{l}4.024 \\
3.980 \\
3.999 \\
3.981\end{array}$ & $\begin{array}{l}11.268 \\
11.014 \\
11.001 \\
11.147\end{array}$ & $\begin{array}{l}65.297 \\
78.82\end{array}$ & $\begin{array}{l}4.450 \\
4.370\end{array}$ \\
\hline$B i_{2} S_{2.75} S e_{0.25}$ & Present (GGA-PBE) & 11.592 & 4.082 & 11.430 & 63.840 & 2.839 \\
\hline
\end{tabular}

\subsection{Electronic properties}

\subsubsection{Electronic band structure}

Based on the optimized lattice parameters, the electronic band structures of $B i_{2} S_{3}$ and $B i_{2} S_{2.75} S_{0.25}$ were evaluated using GGA-PBE approach with and without spin-orbit coupling (SOC). Fig. 3 (a-d) show the electronic band structures plotted along the high symmetry directions for the two structures $B i_{2} S_{3}$ and $B i_{2} S_{2.75} S_{0.25}$. For $B i_{2} S_{3}$, Fig.3a shows an indirect band gap with the valence band maxima (VBM) occurs at the $X$-point whereas the conduction band minima (CBM) is located along the $G-Z$ direction. The calculated band gap of bismuthinite $B i_{2} S_{3}$ at the PBE level is $E_{g}^{P B E}=1.492 \mathrm{eV}$ which is in accordance with other theoretical and experimental results suggesting that $B i_{2} S_{3}$ is an indirect band gap semiconductor ranging from 1.3-1.7 eV [11]. It is well-known that the inclusion of the SOC effect modify the electronic properties of the materials which contain heavy elements. In our case, with the spin-orbit effect consideration, the band gap of $B i_{2} S_{3}$ decreases from $E_{g}^{P B E}=1.492 \mathrm{eV}$ to $E_{g}^{P B E+S O C}=1.208 \mathrm{eV}$. $E_{g}^{P B E+S O C}$ is also an indirect band gap with (VBM) and (CBM) are located at Z-point and $G Y$-path respectively, which is very close to the experimental value of $1.26 \mathrm{eV}$ [27]. The computed energy band gaps of $\mathrm{Bi}_{2} \mathrm{~S}_{3}$ at $\mathrm{PBE}$ and $\mathrm{PBE}+\mathrm{SOC}$ levels are summarized in Table2 within previously theoretical and 
experimental studies. Fig. 3(c-d) shows the electronic band structures of $B i_{2} S_{2.75} S e_{0.25}$ with and without (SOC). Both band structures present a direct band gaps of $E_{g}^{P B E}=1.34 \mathrm{eV}$ and $E_{g}^{P B E+S O C}=1.062 \mathrm{eV}$ at $\mathrm{PBE}$ and $\mathrm{PBE}+\mathrm{SOC}$ level respectively at $\mathrm{Z}$ point. The incorporation of Se atoms in the $B i_{2} S_{3}$ lattice allowed to obtain a narrow band gap leading to activate the intrinsic conduction electrons and subsequently to enhance the electrical transport properties of the undoped $B i_{2} S_{3}$ material. The reduction of the band gap by introducing the Se atoms in $B i_{2} S_{3}$ has been observed experimentally by studying the $\mathrm{Bi}_{2} \mathrm{~S}_{3-\mathrm{x}} \mathrm{Se}_{\mathrm{x}}(\mathrm{x}=0.06-0.75)$ materials [39] in which the band gap passes from $1.35 \mathrm{eV}$ to $1.12 \mathrm{eV}$. Due to the fact that the obtained gap is direct, the $\mathrm{Bi}_{2} \mathrm{~S}_{2.75} \mathrm{Se}_{0.25}$ is satisfactory in optoelectronics and also it can be considered as a light-absorber compound in solar cells due to its value that is close to the optimal value of this kind of materials [40]. The calculated band gaps $E_{g}^{P B E+S O C}$ for $B i_{2} S_{3}$ and the ternary $B i_{2} S_{2.75} S e_{0.25}$ indicate that the (SOC) induces a considerable reduction compared to the $E_{g}^{P B E}$ which can be explained by the splitting states near the Fermi level. 

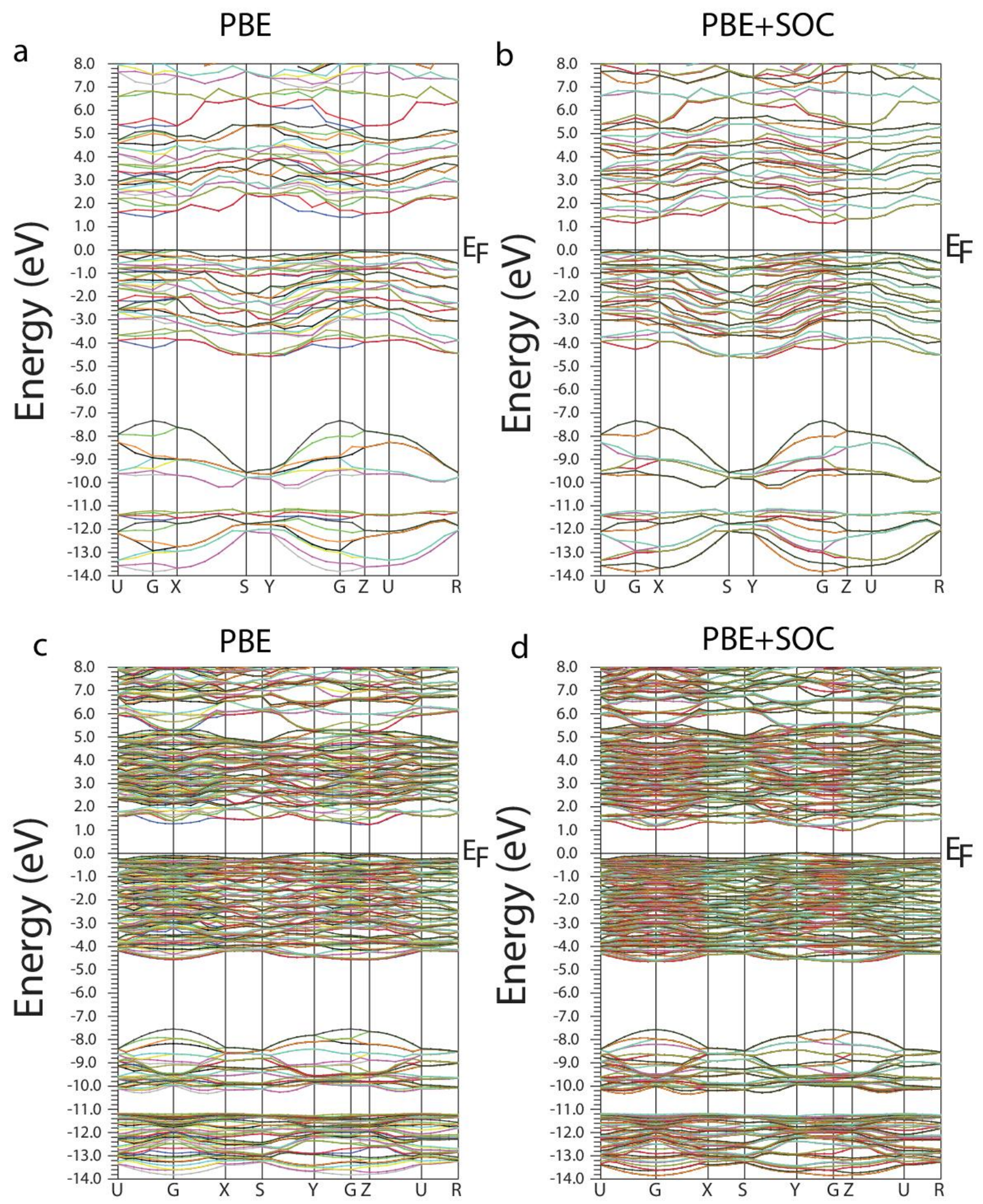

Fig. 3 Calculated electronic band structures of $B i_{2} S_{3}(\mathrm{a}, \mathrm{b})$ and $B i_{2} S_{2.75} S e_{0.25}$ (c,d) with PBE and $\mathrm{PBE}+\mathrm{SOC}$ methods. 
Table 2 The calculated energy band gaps of $\mathrm{Bi}_{2} \mathrm{~S}_{3}$ and $\mathrm{Bi}_{2} \mathrm{~S}_{2.75} \mathrm{Se}_{0.25}$ with PBE and PBE+SOC methods with their corresponding theoretical and experimental data.

\begin{tabular}{lll}
\hline \hline Compound & Method & $\boldsymbol{E}_{\boldsymbol{g}}(\boldsymbol{e V})$ \\
\hline \hline$B i_{2} S_{3}$ & & 1.492 \\
& Present (GGA-PBE) & 1.208 \\
& Present (PBE-SOC) & 1.220 \\
& Theory (DFT-GGA and PAW) [41] & \\
& Theory (LDA-PBE) [27] & 1.380 \\
& Theory (PBE-SOC) [27] & 1.030 \\
& Experimental [27] & 1.260 \\
& Experimental [42] & 1.360 \\
& & 1.340 \\
& & 1.062 \\
\hline
\end{tabular}

\subsubsection{Density of states}

The total density of states (TDOS) and the partial density (PDOS) for $B i_{2} S_{3}$ and $B i_{2} S_{2.75} S_{0.25}$ were calculated to unveil the atom orbital contribution to the band structure. (TDOS) and (PDOS) are calculated with and without (SOC) and are presented in Fig. 4 and Fig. 5. In these figures, the zero energy level is attributed to the Fermi level $\left(E_{f}\right)$. It emerges from Fig. 4 that the lower part of the valence band is dominated by the Bi-6s and S-3s states. The 3p orbitals of S atoms have highly contribution to the upper valence band showing hybridization with Bi-6sp orbitals with a weak presence of $\mathrm{Bi}-5 \mathrm{~d}$ states. The conduction band minimum is composed principally of the $\mathrm{Bi}$ $6 p$ and S-3p characters. The same orbital contributions to the energy bands of $B i_{2} S_{2.75} S e_{0.25}$ are observed with the addition of the $\mathrm{p}$ states of Se atoms which has a considerable contribution in the valence band maximum giving rise to the hybridization with Bi-6p orbitals. The $\mathrm{Se} 4 \mathrm{p}$ states contribute also in the conduction band minimum besides the $\mathrm{p}$ states of $\mathrm{Bi}$ and $\mathrm{S}$ atoms. After the inclusion of the (SOC), the contribution of the different states in the valence and the conduction 
bands remains unchanged but a remarkable reduction in their densities is observed as shown in the Fig 4(d) and Fig 5(d) for the two materials. We find also that the bandwidth has increase by 0.112 $\mathrm{eV}$ for $\mathrm{Bi}_{2} \mathrm{~S}_{3}$ and by $0.086 \mathrm{eV}$ for $B i_{2} S_{2.75} S_{0.25}$ in the presence of (SOC). The obtained results from the electronic band structures as well as the density of states prove the influence of the introduction of the (SOC) on the electronic structure of the undoped and doped bismutinite $B i_{2} S_{3}$.
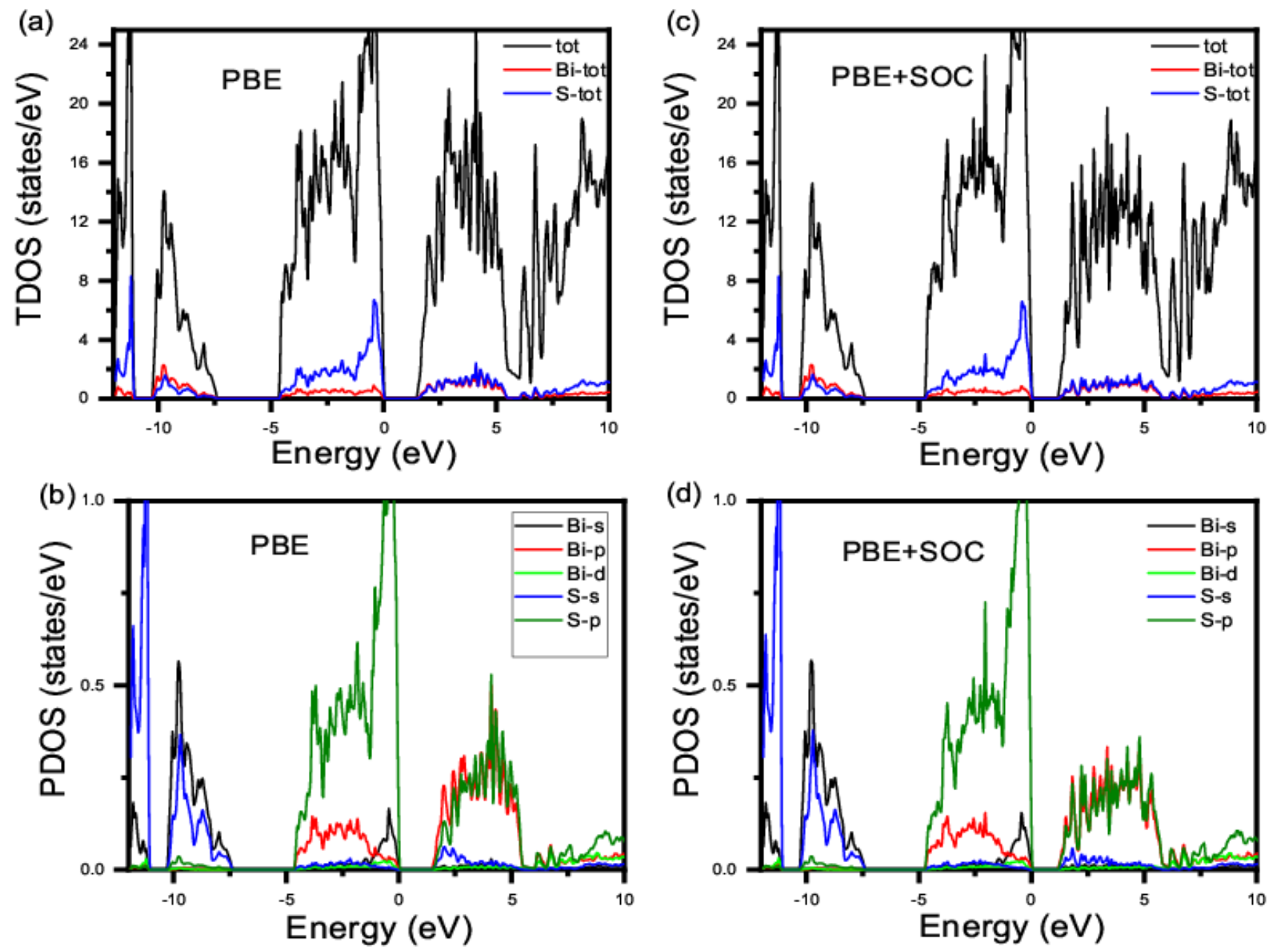

Fig. 4 Calculated total (TDOS) and partial density of states (PDOS) of $B i_{2} S_{3}$ with PBE and PBE+SOC methods. 

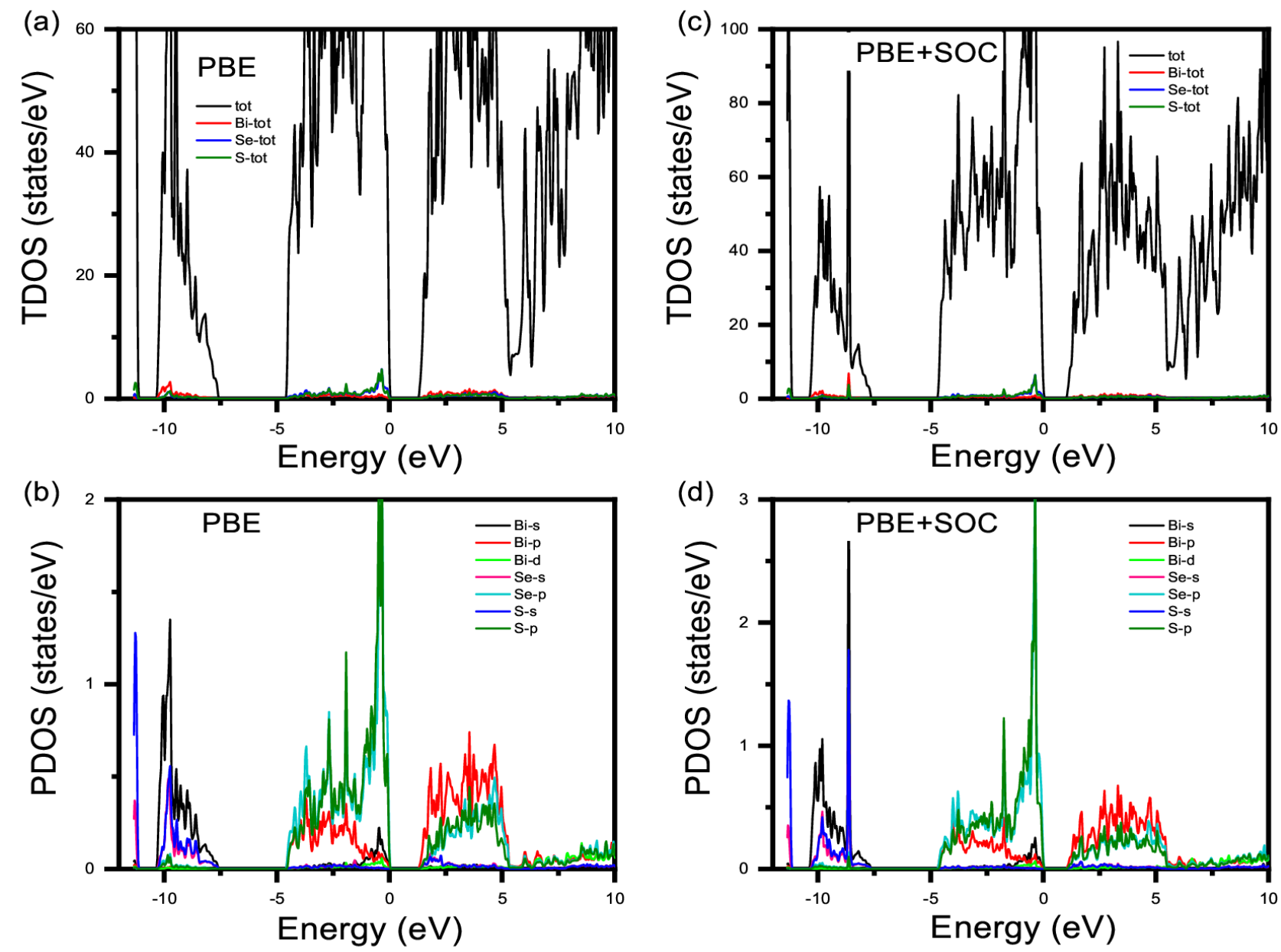

Fig. 5 Calculated total (TDOS) and partial density of states (PDOS) of $B i_{2} S_{2.75} S e_{0.25}$ with PBE and PBE+SOC methods.

\section{2. 3 Effective masses}

To understand the physical mechanism of the thermoelectric property of bismuthinite and the Sedoped bismuthinite $\left(B i_{2} S_{3}\right)$, carrier effective masses were calculated at $G$-point along $G-Z$ $(0,0,1 / 2)$ and $G-X(1 / 2,0,0)$ directions in the first Brillouin zone of the pbnm orthorhombic structure. For the calculation of the effective masses we use the approximation model given by [43] based on the calculated band structures with PBE+SOC model. The results are depicted in Table 3. As it is presented in Table 3, the electron effective masses $m_{e}^{*}$ for both $B i_{2} S_{3}$ and $B i_{2} S_{2.75} S e_{0.25}$ are smaller than the effective hole masses $m_{h}^{*}$, which is in accordance with the 
experimental and theoretical studies [27, 41]. For $B i_{2} S_{3}$, our calculated average value of $m_{e}^{*}$ is $1.85 m_{0}$ consistent with the previous experimental data [44] showing that $m_{e}^{*}$ is ranging from 0.6 to $2.2 m_{0}$. The carrier effective mass anisotropy can be attributed to the anisotropic ratio $\frac{m_{c}^{*}}{m_{a}^{*}}$ where $m_{c}^{*}$ and $m_{a}^{*}$ are the carrier effective masses along the $(G-Z)$ and $(G-X)$ directions respectively. The calculated electron effective mass anisotropy is 1.30 for $B i_{2} S_{3}$ and 2.42 for $B i_{2} S_{2.75} S_{0.25}$. The obtained higher anisotropy is related to the chemical bonds in the two crystals. The anisotropy increased when selenium atoms were substituted in $B i_{2} S_{3}$ crystal resulting in a more covalent bonds along c-axis and a weaker ionic bonds along the $\mathrm{a}$ and $\mathrm{b}$ directions. In addition, the introduction of the Se atoms in bismuthinite crystal has the effect of reducing the electron and hole effective masses and thus an increase in the carrier mobility. This has been observed experimentally by Ajara et al. [28].

Table 3 Calculated effective masses of electrons and holes in units of free electron mass $m_{0}$ for $B i_{2} S_{3}$ and $B i_{2} S_{2.75} S e_{0.25}$ along $G-X$ and $G-Z$ directions.

\begin{tabular}{cccc}
\hline System & Direction & $m_{e}^{*}\left[m_{0}\right]$ & $m_{h}^{*}\left[m_{0}\right]$ \\
\hline \hline$B i_{2} S_{3}$ & $G-Z$ & 2.099 & 3.850 \\
& $G-X$ & 1.607 & 4.530 \\
$\mathrm{Bi}_{2} \mathrm{~S}_{2.75} \mathrm{Se}_{0.25}$ & $G-Z$ & 0.489 & 1.301 \\
& $G-X$ & 1.187 & 2.110 \\
\hline \hline
\end{tabular}

\section{3 Optical properties}

\section{3. 1 Linear optical properties}

The linear optical properties of $B i_{2} S_{3}$ and $B i_{2} S_{2.75} S_{0.25}$ are calculated with and without (SOC) for radiation up to $12 \mathrm{eV}$ as shown in Figs 6-10. All optical parameters are derived from the complex dielectric function $\epsilon(\omega)=\epsilon_{1}(\omega)+i \epsilon_{2}(\omega)$ which define the optical response of a medium at all photon energy. $\epsilon_{1}(\omega)$ is the real part of $\epsilon(\omega)$ corresponds to the dispersive behavior of the material while $\epsilon_{2}(\omega)$ is the imaginary part of $\epsilon(\omega)$ describing the absorptive behavior of 
the medium [45]. As $B i_{2} S_{3}$ and $B i_{2} S_{2.75} S e_{0.25}$ crystallize in orthorhombic structure, the dielectric tensor $\epsilon(\omega)$ have three diagonal independent components $\left(\epsilon_{x x}, \epsilon_{y y}, \epsilon_{z z}\right)$. In this paper, we have restricted our study of the optical properties for polarization parallel to the $\mathrm{z}$ direction $(E \| z)$ which is considered as a preferential direction for enhancing electro-optical properties of $B i_{2} S_{3}$ [46]. $\epsilon_{1}(\omega)$ spectra calculated with and without (SOC) for $B i_{2} S_{3}$ and $B i_{2} S_{2.75} S e_{0.25}$ for $(E \| z)$ are given by Fig. 6 .
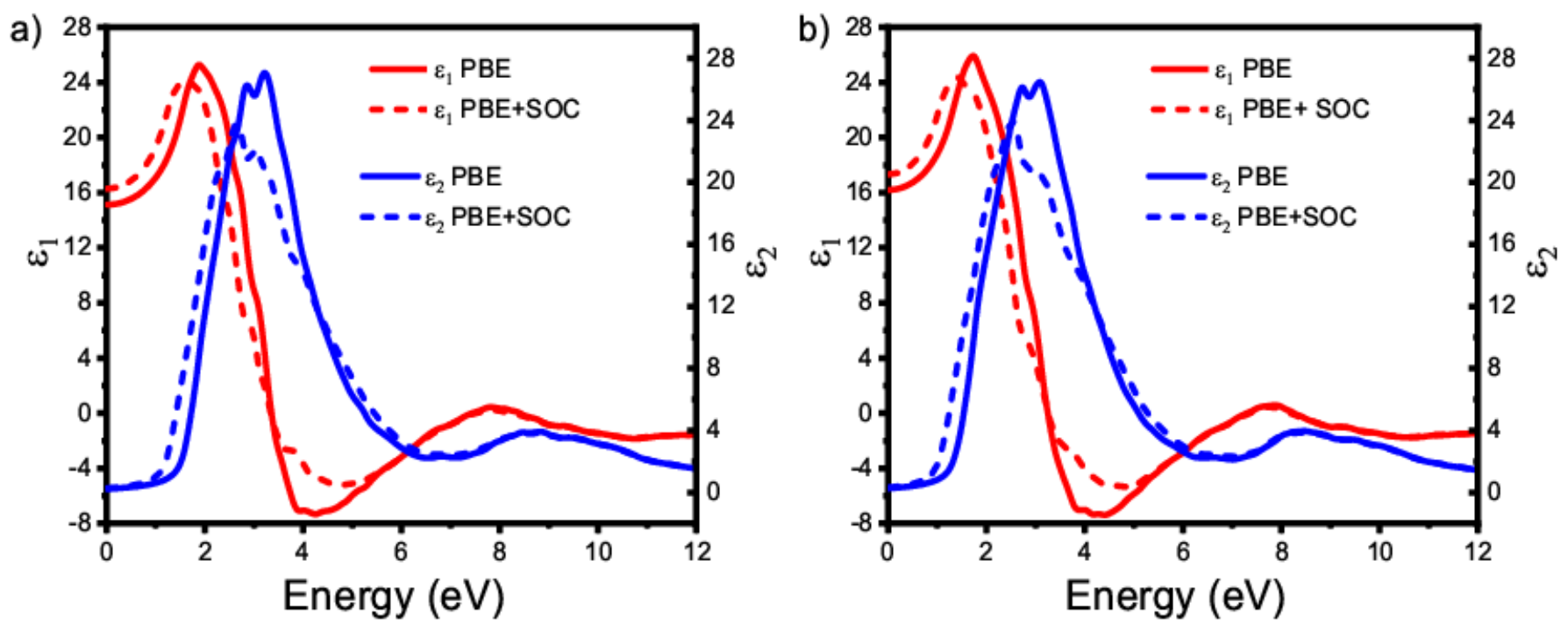

Fig. 6 Calculated real and imaginary parts of the dielectric function of $B i_{2} S_{3}$ (a) and $B i_{2} S_{2.75} S e_{0.25}$ with PBE and PBE+SOC method.

All $\epsilon_{1}(\omega)$ curves start from the static dielectric constant $\epsilon_{1}(0)$ which corresponds to the dielectric response at zero frequency. The static dielectric constants for $B i_{2} S_{3}$ and $B i_{2} S_{2.75} S_{0.25}$ are listed in Table 4. It can be seen from Table 4 that $\epsilon_{1}(0)$ increased with the addition of the Se element and in the presence of the (SOC) giving rise to a high dielectric constant. To study the stability of the excitons, we calculate the exciton binding energy which can be calculated using the Bohr model [47],

$$
E_{b}=\frac{13.6 \mathrm{eV} \cdot \mu^{*}}{\epsilon_{\infty}(0)^{2}}
$$

where $\mu^{*}$ is the reduced mass given by : $\mu^{*}=\frac{m_{e}^{*} m_{h}^{*}}{\left(m_{e}^{*}+m_{h}^{*}\right)}$ and $\epsilon_{\infty}(0)$ is the static dielectric constant computed without (SOC) and listed in Table 4 . To evaluate $\mu^{*}$, we considered the directionally 
averaged value for $m_{e}^{*}$ and $m_{h}^{*}$ given from the Table 3. Our calculated binding energy $E_{b}$ for $B i_{2} S_{3}$ and $B i_{2} S_{2.75} S e_{0.25}$ are $74.16 \mathrm{meV}$ and $29.10 \mathrm{meV}$ respectively. The calculated values of $E_{b}$ are in the same order of that of II-VI compounds [48]. The obtained results for $E_{b}$ indicate that the incorporation of selenium ( $\mathrm{Se}$ ) atoms in the bismithinite $B i_{2} S_{3}$ decreases the exciton binding energy which induces a reduction in recombination, thus improving the efficiency of the extraction of the charge carriers.

Table 4 Calculated optical constants for $B i_{2} S_{3}$ and $B i_{2} S_{2.75} S_{0.25}$ : static dielectric constant $\epsilon_{1}(0)$ , static refractive index $n(0)$, oscillator energy $E_{0}$, dispersion energy $E_{d}$ and oscillator strength $\begin{array}{llll}\mathcal{F} \text { calculated with and without } & \text { SOC. }\end{array}$

\begin{tabular}{clccccc}
\hline \hline Compound & Method & $\boldsymbol{\epsilon}_{\mathbf{1}}(\mathbf{0})$ & $\boldsymbol{n}(\mathbf{0})$ & $\boldsymbol{E}_{\mathbf{0}}(\boldsymbol{e V})$ & $\boldsymbol{E}_{\boldsymbol{d}}(\boldsymbol{e V})$ & $\mathcal{F}\left(\boldsymbol{e V ^ { 2 }}\right)$ \\
\hline \hline$B i_{2} S_{3}$ & Present (GGA-PBE) & 14.981 & 3.886 & 2.866 & 40.429 & 115.869 \\
& Present (PBE-SOC) & 16.183 & 3.887 & 2.135 & 30.122 & 54.3100 \\
$B_{2} S_{2.75} S_{0.25}$ & Present (GGA-PBE) & 16.315 & 4.310 & 2.268 & 39.882 & 104.809 \\
& Present (PBE-SOC) & 17.375 & 4.157 & 2.214 & 36.061 & 79.8390
\end{tabular}

It can be seen from Fig. $6(\mathrm{a}-\mathrm{b})$, that $\epsilon_{1}(\omega)$ decrease to values below zero indicating that the reflection is maximum in the energy range about (3-7) $\mathrm{eV}$ and the materials exhibit metallic behavior in this area. The absorptive part $\epsilon_{2}(\omega)$ calculated with and without (SOC) for $(E \| z)$ is illustrated in Fig. 6 (a-b) for both compounds. $\epsilon_{2}(\omega)$ spectra represent a pronounced peak located at $3.233 \mathrm{eV}$ and $3.102 \mathrm{eV}$ for $B i_{2} S_{3}$ and $B i_{2} S_{2.75} S e_{0.25}$ respectively before the inclusion of the (SOC). With the inclusion of the (SOC), these high intensity peak of $\epsilon_{2}$ show a red shift. A second peak, who is weaker than the first one, appears at $8.861 \mathrm{eV}$ and $8.512 \mathrm{eV}$ before and after inclusion of the (SOC) for $B i_{2} S_{3}$ and $B i_{2} S_{2.75} S e_{0.25}$ respectively. Thus, these differences in the results of the dielectric constants confirm the crucial role of SOC effects in the optical properties of undoped and doped $B i_{2} S_{3}$. Our calculated maximum value of $\epsilon_{2}$ for $(E \| z)$ for $B i_{2} S_{3}$ is in accordance with maximum peak values given theoretically by [49]. It is known that $\epsilon_{2}(\omega)$ is directly related to the 
optical electron transition. From PDOS displayed in Figs. 4-5 and for both compounds, the transition between the occupied states Bi 6s to unoccupied states $\mathrm{S} 3 \mathrm{p}$ is at the origin of the appearance of the main peaks, while the second peaks originate from transition occurring between Bi 6s and S 3p electrons. For $B i_{2} S_{2.75} S e_{0.25}$ there is another transition between Se 3p and Bi $6 \mathrm{p}$ states participating in the appearance of the main peak in $\epsilon_{2}(\omega)$.

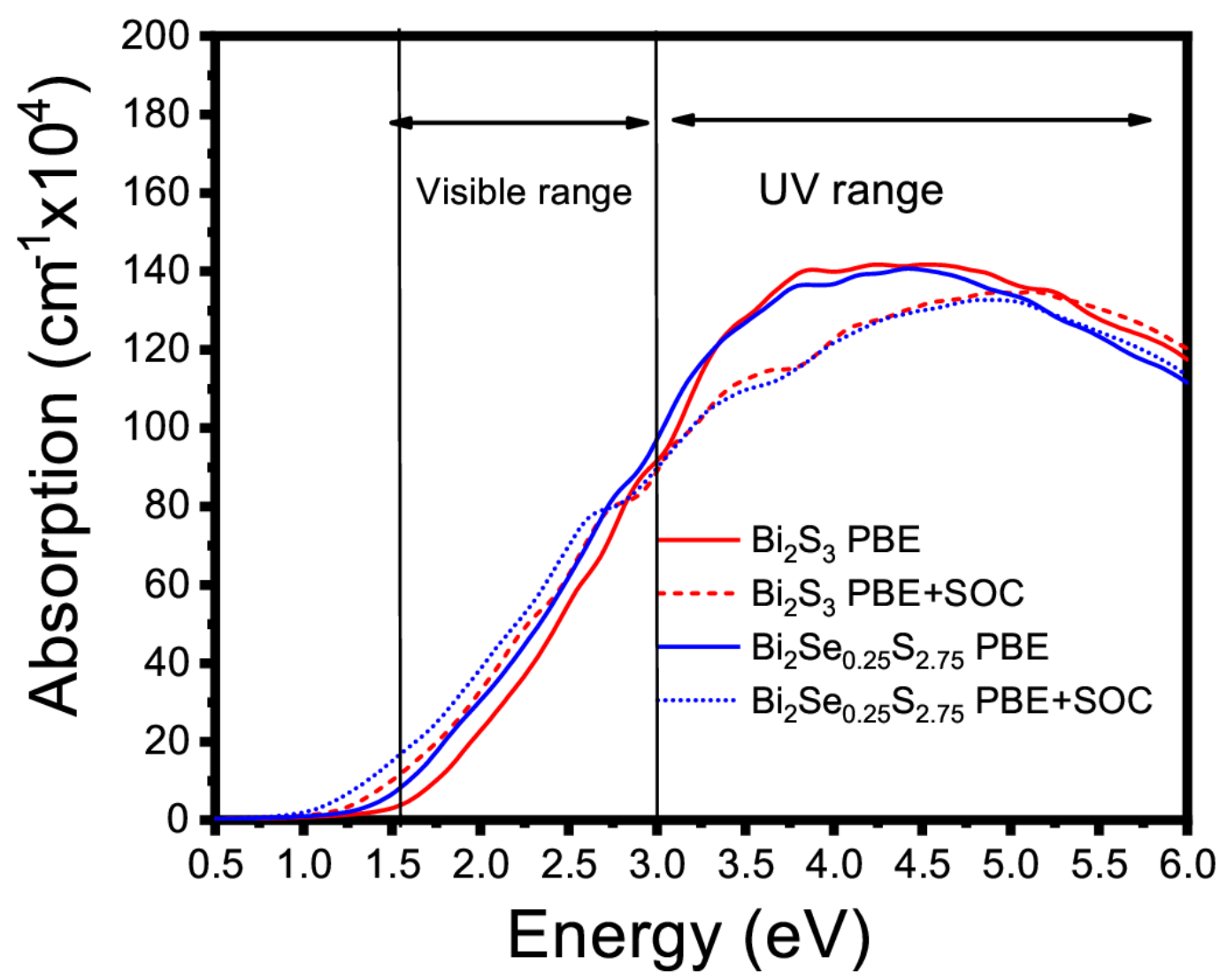

Fig. 7 Calculated absorption coefficient $\alpha(\omega)$ of $B i_{2} S_{3}$ and $B i_{2} S_{2.75} S_{0.25}$ with PBE and PBE+SOC methods.

The absorption coefficient $\alpha(\omega)$ spectra for $(E \| z)$ calculated with and without (SOC) for $B i_{2} S_{3}$ and $\mathrm{Bi}_{2} \mathrm{~S}_{2.75} \mathrm{Se}_{0.25}$ are given by Fig. 7. As can be seen from the $\alpha(\omega)$ spectra, the threshold of absorption is around $1.391 \mathrm{eV}$ and $1.305 \mathrm{eV}$ for undoped and doped $B i_{2} S_{3}$ respectively. This shows 
that there was a shift towards low energies on the absorption edge with the incorporation of the Se atoms. This confirms the experimental results carried out on $\mathrm{Bi}_{2} \mathrm{~S}_{3-\mathrm{x}} \mathrm{Se}_{\mathrm{x}}$ [27]. With the addition of (SOC), the threshold of absorption decrease and take the values of $1.192 \mathrm{eV}$ and $1.010 \mathrm{eV}$ for $\mathrm{Bi}_{2} \mathrm{~S}_{3}$ and $\mathrm{Bi}_{2} \mathrm{~S}_{2.75} \mathrm{Se}_{0.25}$ respectively. The calculated energies of the threshold of absorption are close to the energy band gaps of $B i_{2} S_{3}$ and $B i_{2} S_{2.75} S e_{0.25}$ evaluated with and without the addition of (SOC) (see Table 2). In the energy range up to $3 \mathrm{eV}$, the absorption is maximum for $B i_{2} S_{2.75} S e_{0.25}$ has a value of $94.79310^{4} \mathrm{~cm}^{-1}$, while at the same energy the undoped bismuthinite present an absorption of the order of $90.22810^{4} \mathrm{~cm}^{-1}$ in agreement with the experimental values $\left(104-10510^{4} \mathrm{~cm}^{-1}\right)$ [10]. This clearly shows that $B i_{2} S_{3}$ becomes more absorbent in the visible range by doping it with selenium. For higher energy extending up to $3 \mathrm{eV}$, $B i_{2} S_{3}$ crystal has a higher absorption than that of $B i_{2} S_{2.75} S e_{0.25}$. The calculated high absorption coefficient of the ternary $B i_{2} S_{2.75} S e_{0.25}$ shows that it is a very promising material in photovoltaic applications. The addition of the (SOC) acts strongly on the absorption spectra particularly in the visible range where a slight shift is observed for both compounds. The optical conductivity $\sigma(\omega)$ describes the electron conduction produced by an incident photon crossing a material. The optical conductivity spectra for $(\mathrm{E} \| \mathrm{z})$ calculated with and without addition of the (SOC) for both compounds are presented in Fig. 8. The optical conductivity presents a sharp peak in the visible range for both compounds $B i_{2} S_{3}$ and $B i_{2} S_{2.75} S e_{0.25}$. The maximum value of the optical conductivity decrease in the case of the calculation with (SOC) effect. $L(\omega)$ is the electron energy loss function describing the energy loss of a fast electron crossing a medium. The energy loss (Eloss) spectra for $B i_{2} S_{3}$ and $B i_{2} S_{2.75} S e_{0.25}$ calculated with and without (SOC) for (E\|z) are given by Fig. 9. L $(\omega)$ present a prominent peak called the plasma resonance [50] located around 7.231 $\mathrm{eV}$ and $7.138 \mathrm{eV}$ for $\mathrm{Bi}_{2} S_{3}$ and $B i_{2} S_{2.75} S e_{0.25}$ materials respectively. The corresponding plasma frequencies are $10.980 \times 10^{15} \mathrm{~Hz}$ and $10.839 \times 10^{15} \mathrm{~Hz}$. The calculated value of the plasma excitations for $B i_{2} S_{3}$ is in accordance with theoretical results [49] and experimental measurements [51].

The refractive index $n(\omega)$ and the extinction coefficient $k(\omega)$ for $(E \| z)$ are plotted in the Fig. 10. It can be seen that $n(\omega)$ curves present an important value in low energy region and remarkable reduction in high energy region. To analyze the refractive index at lower optical frequencies one can use the Wemple - DiDomenico (WDD) single-oscillator model [52] which is the most applied 
concept for the chalcogenide semiconductors. The (WDD) model is given by the following expression:

$$
\left(n^{2}-1\right)^{-1}=\frac{E_{0}}{E_{d}}-\frac{1}{E_{0} E_{d}}(h v)^{2}
$$

here, $E_{0}$ and $E_{d}$ are the dispersion energy parameters, h is Planck's constant and hv is the photon energy.

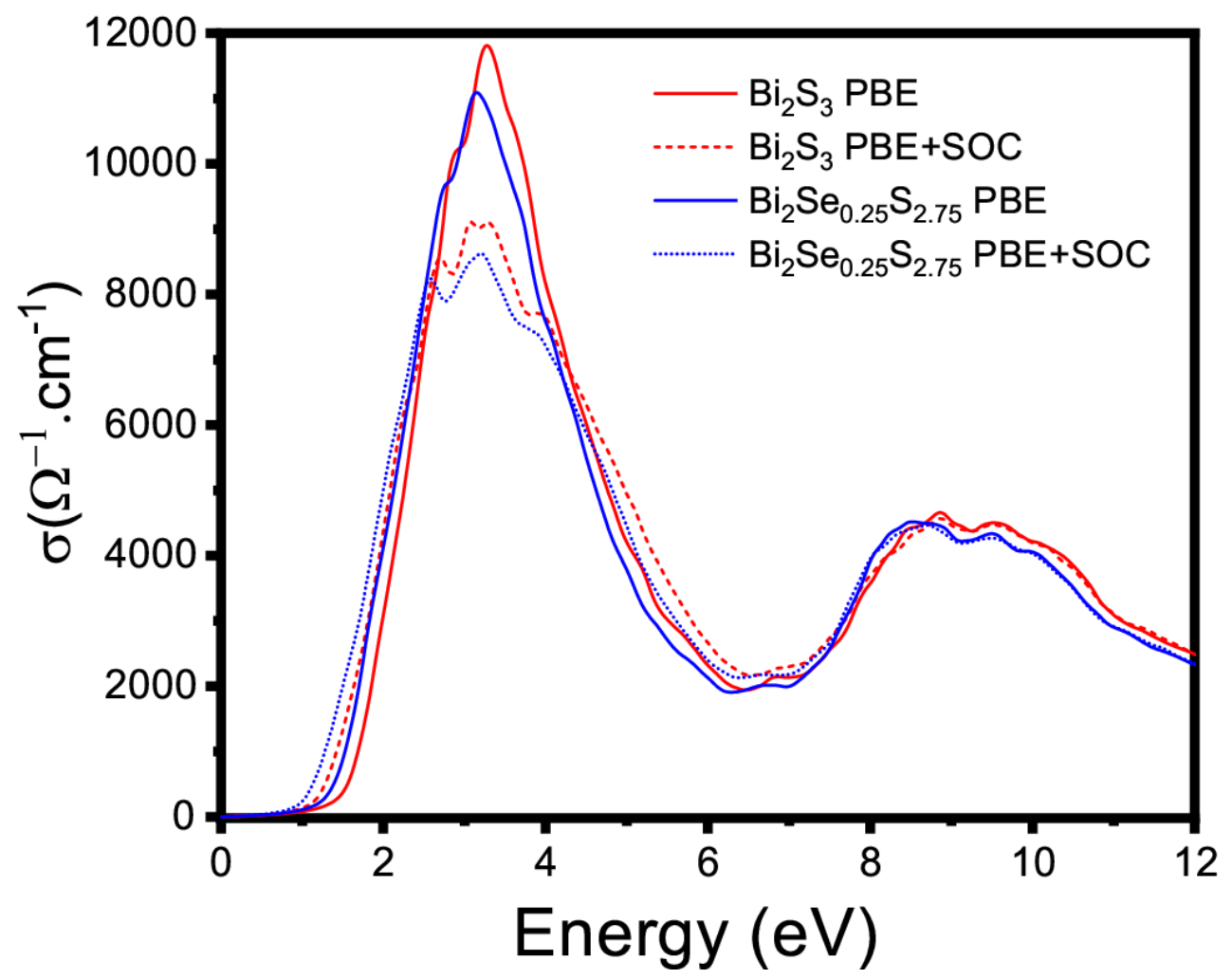

Fig. 8 Calculated optical conductivity $\sigma(\omega)$ of $B i_{2} S_{3}$ and $B i_{2} S_{2.75} S e_{0.25}$ with PBE and PBE+SOC methods. 


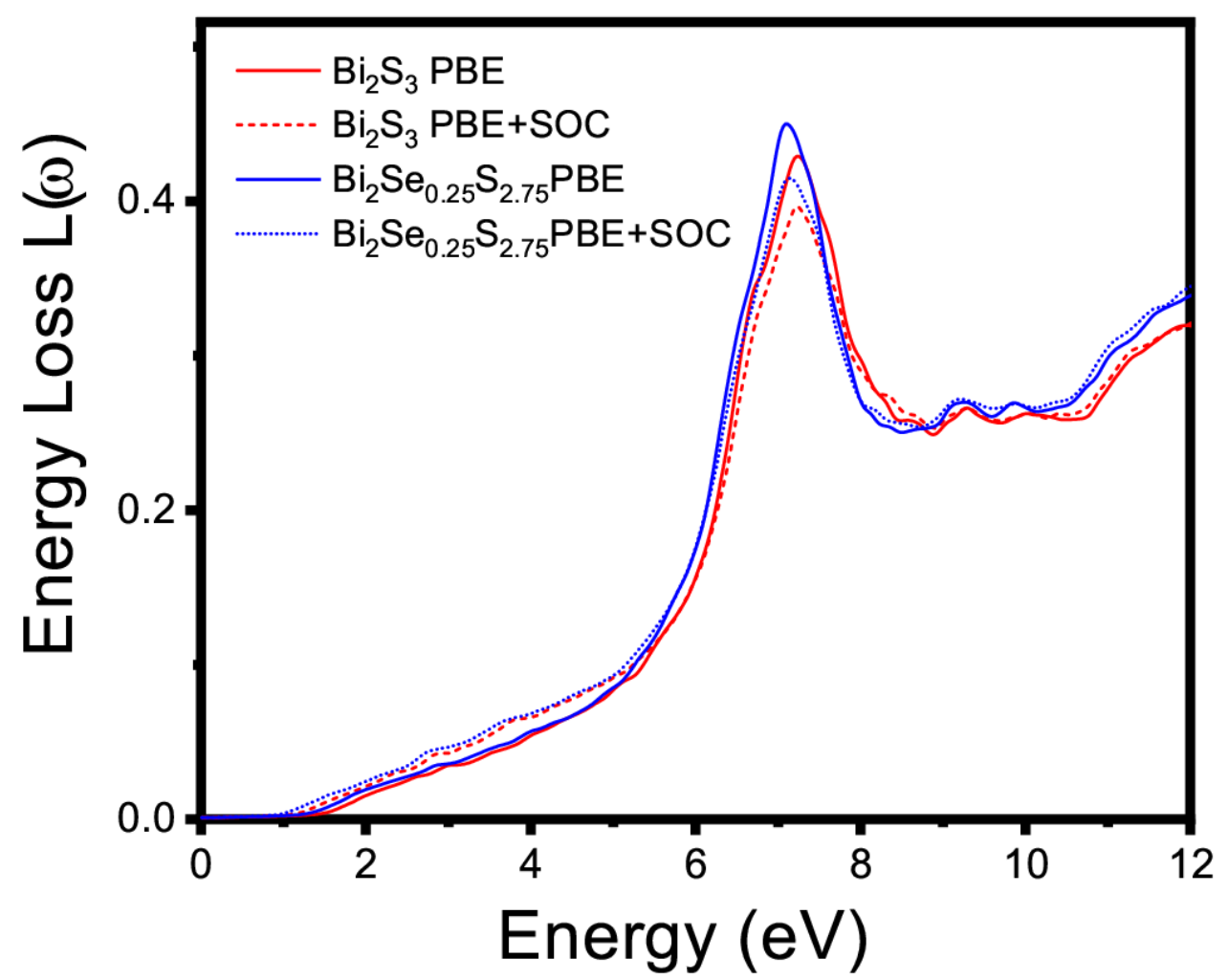

Fig. 9 Calculated energy loss function $\mathrm{L}(\omega)$ of $B i_{2} S_{3}$ and $B i_{2} S_{2.75} S e_{0.25}$ with PBE and PBE+SOC methods.

a)

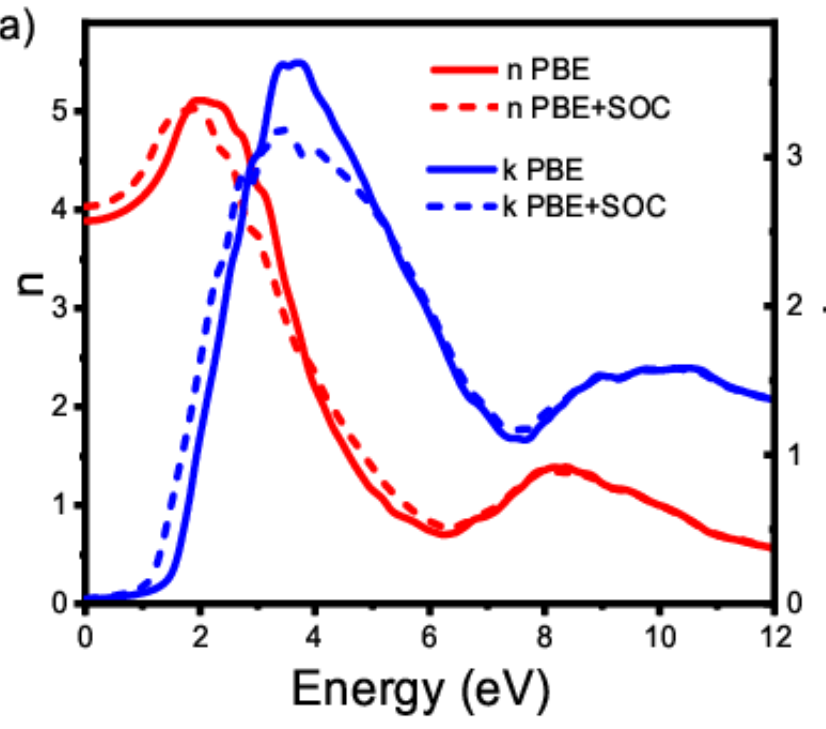

b)

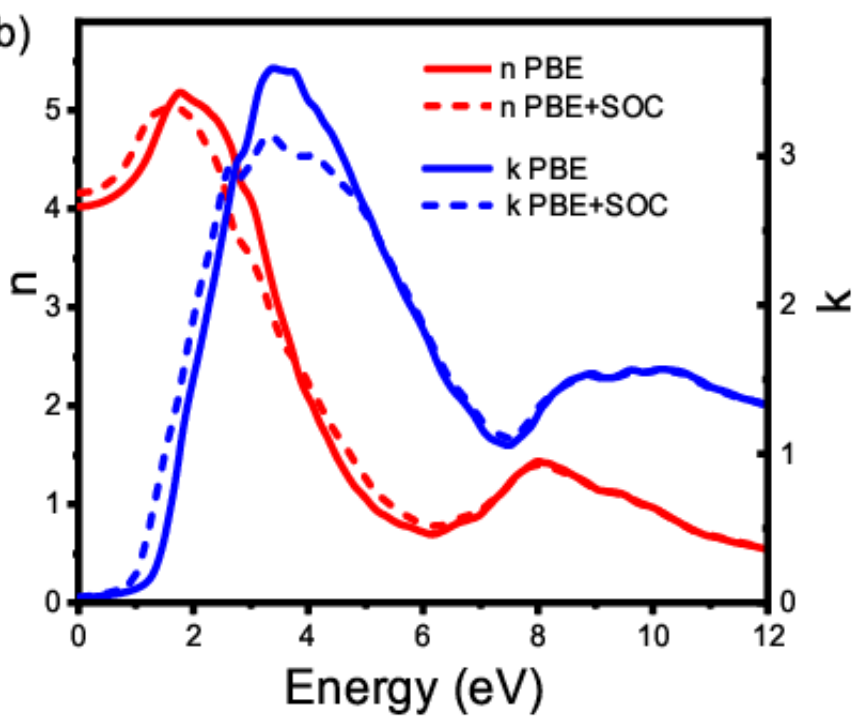

Fig. 10 Calculated complex refractive index $n(\omega)$ and extinction coefficient $k(\omega)$ of $B i_{2} S_{3}$ (a) and $\mathrm{Bi}_{2} \mathrm{~S}_{2.75} \mathrm{Se}_{0.25}$ (b) with PBE and PBE+SOC methods. 
The dispersion energy $E_{d}$ corresponds to the dispersion of the electronic dielectric function related to the interband transition force, while $E_{0}$ is the single oscillator energy connected with the optical energy gap called WDD band gap [52]. The dispersion parameters for $B i_{2} S_{3}$ and $B i_{2} S_{2.75} S e_{0.25}$ were estimated by fitting the plot $\left(n^{2}-1\right)^{-1}$ versus $(h v)^{2}$ in low energy region as shown in Fig. 11. Our calculated values of $E_{d}$ and $E_{0}$ for $B i_{2} S_{3}$ and $B i_{2} S_{2.75} S e_{0.25}$ calculated with and without (SOC) are listed in Table 4. From Table 4, the dispersion energy $E_{d}$ decreased while passing from $B i_{2} S_{3}$ and $B i_{2} S_{2.75} S e_{0.25}$ after and before the inclusion of (SOC). This is essential due to the addition of the Se atoms in $B i_{2} S_{3}$ lattice which induced a weak force of the interband transitions. Similarly, the oscillatory energy $E_{0}$ decreases for $B i_{2} S_{2.75} S e_{0.25}$ due to the decrease in the energy band gap. Based on the (WDD) model, we can calculate the static refractive index $n(0)$ from

equation: $n(0)=\sqrt{1+\left(\frac{E_{d}}{E_{0}}\right)}$. The static refractive indexes $n(0)$ for $B i_{2} S_{3}$ and $B i_{2} S_{2.75} S e_{0.25}$ calculated with and without (SOC) are given in Table 4. Another important parameter given by the WDD model is the oscillator strength given by $: \mathcal{F}=E_{0} E_{d}$. The calculated values of $\mathcal{F}$ for $B i_{2} S_{3}$ and $B i_{2} S_{2.75} S e_{0.25}$ are reported in Table 4.

\subsubsection{Non-linear optical parameters}

The optical non-linearity of materials is directly related to the control of the light in optical switching devices. In the present work, the nonlinear optical properties (NLO) were studied from the calculation of the 3 rd order nonlinear optical susceptibility $\chi^{(3)}$ and the non-linear refractive index $n_{2}$ for $B i_{2} S_{3}$ and $B i_{2} S_{2.75} S e_{0.25}$ within the Miller's rule and the static refractive index $n(0)$ [52]. It is known that the non-linearity occurs when the electromagnetic field becomes high enough. $\chi^{(3)}$ can be evaluated by the following expression: 


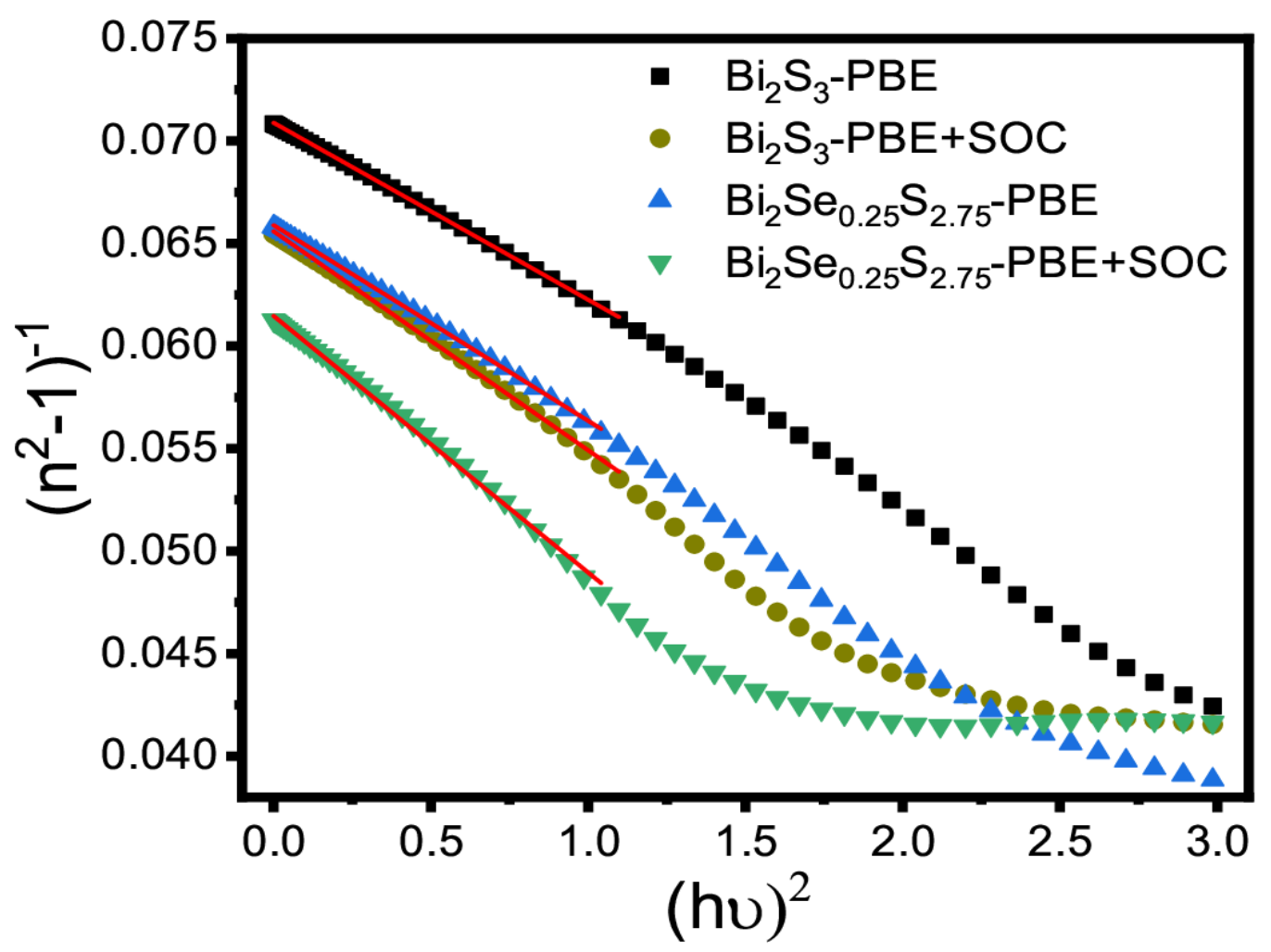

Fig. $11\left(n^{2}-1\right)^{-1}$ versus $(h v)^{2}$ for $B i_{2} S_{3}$ and $B i_{2} S_{2.75} S e_{0.25}$ plotted with and without SOC

$$
\chi^{(3)}=\frac{A}{(4 \pi)^{4}}\left(\frac{E_{d}}{E_{0}}\right)^{4}
$$

where $\mathrm{A}$ is a constant independent of the frequency and equal to $1.7 \times 10^{-10} \mathrm{esu}$ [52], $E_{d}$ and $E_{0}$ are the dispersion parameters given by the (WDD) single-oscillator model. The non-linear refractive index $n_{2}$ is related to $\chi^{(3)}$ and the static refractive index $n_{0}$ and given by:

$$
n_{2}=\frac{12 \pi \chi^{(3)}}{n(0)}
$$

The calculated values of $\chi^{(3)}$ and $n_{2}$ for $B i_{2} S_{3}$ and $B i_{2} S_{2.75} S e_{0.25}$ are summarized in Table 5. 
Table 5 Non-linear optical parameters: third-order nonlinear optical susceptibility $\chi^{(3)}$ and nonlinear refractive index $n_{2}$ for $B i_{2} S_{3}$ and $B i_{2} S_{2.75} S e_{0.25}$ calculated with and without (SOC).

\begin{tabular}{clll}
\hline \hline Compound & Method & $\chi^{(\mathbf{3})} \mathbf{1 0}^{-\mathbf{1 0}}$ esu & $\boldsymbol{n}_{\mathbf{2}} \mathbf{1 0}^{-\mathbf{1 0}}$ esu \\
\hline \hline & & & \\
$\mathrm{Bi}_{2} S_{3}$ & Present (GGA-PBE) & 2.699 & 4.363 \\
& Present (PBE-SOC) & 2.701 & 4.369 \\
& & & \\
$\mathrm{Bi}_{2} \mathrm{~S}_{2.75} \mathrm{Se}_{0.25}$ & Present (GGA-PBE) & 6.512 & 9.493 \\
& & & \\
& Present (PBE-SOC) & 4.797 & 7.493
\end{tabular}

From Table 5, the calculated values of the (NLO) parameters $\chi^{(3)}$ and $n_{2}$ for $B i_{2} S_{3}$ and $B i_{2} S_{2.75} S e_{0.25}$ are large and this can be related to the electronic and geometrical structure of these two materials. The obtained value of $\chi^{(3)}$ coefficient of the bulk $B i_{2} S_{3}$ is larger than measured experimentally for $B i_{2} S_{3}$ nanocrystal: $6.25 \times 10^{-11}$ esu [53]. This difference is due in particular to the quantum confinement effect in the nanocrystals. In addition, the results presented in Table 5 show that doping $B i_{2} S_{3}$ with Se atoms further expanded the (NLO) coefficients $\chi^{(3)}$ and $n_{2}$ thus showing the effect of Se doping $B i_{2} S_{3}$ in improving the nonlinear optical properties and consequently in the realization of a promising nonlinear optical materials.

\section{Conclusion}

The effect of spin orbit coupling on electronic and optical properties of $B i_{2} S_{2.75} S e_{0.25}$ are investigated by means of Wien $2 \mathrm{k}$ code based on the density functional theory. Firstly, the structural properties such as lattice constants, the bulk modulus and its pressure derivative of $B i_{2} S_{3}$ and $\mathrm{Bi}_{2} S_{2.75} S e_{0.25}$ are calculated within the PBE-GGA scheme. The calculated electronic band structures show that $B i_{2} S_{2.75} S e_{0.25}$ is a direct band gap semiconductor with and without the inclusion of (SOC). The energy band gap reduction with respect to that of undoped $B i_{2} S_{3}$ is due 
to the splitting states near the Fermi level when the (SOC) is taken into account. The carrier effective masses of $\mathrm{Bi}_{2} S_{3}$ and $\mathrm{Bi}_{2} S_{2.75} S e_{0.25}$ were calculated along high symmetry directions showing an increase in the anisotropy when Se atoms were incorporated and an increase in the carrier mobility. The linear optical properties of $B i_{2} S_{3}$ and Se doped $B i_{2} S_{3}$ (dielectric function, absorption, optical conductivity and energy loss function) were investigated for polarization parallel to the $\mathrm{z}$ axis. All linear optical spectra show a red shift when incorporating Se atoms in $B i_{2} S_{3}$ lattice and after inclusion of (SOC). $B i_{2} S_{2.75} S e_{0.25}$ exhibits a high absorption coefficient in the visible region showing its potential use in future for applications in solar cells. The static dielectric constant increase in $B i_{2} S_{2.75} S e_{0.25}$ with (SOC) effect giving rise to a decrease in the exciton binding energy. The oscillator parameters : the oscillator energy, the dispersion energy and the static refractive index for both $B i_{2} S_{3}$ and $B i_{2} S_{2.75} S e_{0.25}$ materials were estimated using the (WDD) single-oscillator model. It was found that the inclusion of Se content in $B i_{2} S_{3}$ leads to the increase of the static refractive index but a decrease in the oscillator parameters. The nonlinear optical properties (NLO) of doped and undoped $\mathrm{Bi}_{2} \mathrm{~S}_{3}$ were also reported. A large values of (NLO)

parameters were obtained for $B i_{2} S_{2.75} S e_{0.25}$ indicating that it can be a useful device for all-optical communication and computer network. The present work constitutes a first theoretical report about the substitution of the selenium atoms for sulfur sites in $B i_{2} S_{3}$. We strongly believe that our study on the electronic, linear and nonlinear optical properties of the ternary bismuth sulfide $B i_{2} S_{2.75} S e_{0.25}$ is important for the development of a new high-performance optoelectronic materiel in the Bi-based compounds.

\section{CrediT authors statement}

Houda Ben Abdallah: Formal analysis, Methodology, Investigation, Writing-original draft, Supervision, Validation, Conceptualization Walid Ouerghui: Formal analysis, Validation, Investigation, Conceptualization, Writing - review \& editing.

\section{Declaration of competing interests}

The authors declare that they have no known competing financial interests or personal relationships that could have appeared to influence the work reported in this paper. 


\section{REFERENCES}

1. Koc, H., Ozisik, H., Deligöz, E., Mamedov, A.M., Ozbay, E.: Mechanical, electronic, and optical properties of $\mathrm{Bi} 2 \mathrm{~S} 3$ and Bi2Se3 compounds: First principle investigations. J. Mol. Model. 20, 2180 (2014). https://doi.org/10.1007/s00894-014-2180-1

2. Ganose, A.M., Butler, K.T., Walsh, A., Scanlon, D.O.: Relativistic electronic structure and band alignment of BiSI and BiSeI: Candidate photovoltaic materials. J. Mater. Chem. A. 4, 2060-2068 (2016). https://doi.org/10.1039/c5ta09612j

3. Deng, J., Zhao, Z.Y.: Electronic structure and optical properties of bismuth chalcogenides Bi2Q3 (Q = O, S, Se, Te) by first-principles calculations. Comput. Mater. Sci. 142, 312319 (2018). https://doi.org/10.1016/j.commatsci.2017.10.032

4. Konstantatos, G., Levina, L., Tang, J., Sargent, E.H.: Sensitive solution-processed Bi2S3 nanocrystalline photodetectors. Nano Lett. 8, 4002-4006 (2008).

https://doi.org/10.1021/n1802600z

5. Harman, T.C., Taylor, P.J., Walsh, M.P., LaForge, B.E.: Quantum dot superlattice thermoelectric materials and devices. Science (80-. ). 297, 2229-2232 (2002). https://doi.org/10.1126/science.1072886

6. Kim, J.H., Lim, T., Park, J.Y., Ma, A., Jung, H., Kim, H.Y., Cho, S.K., Yoon, H., Nam, K.M.: Understanding and improving photoelectrochemical performance of $\mathrm{Bi} 2 \mathrm{O} 3 / \mathrm{Bi} 2 \mathrm{~S} 3$ composite. New J. Chem. 43, 11893-11902 (2019). https://doi.org/10.1039/c9nj02913c

7. Wu, T., Zhou, X., Zhang, H., Zhong, X.: Bi2S3 nanostructures: A new photocatalyst. Nano Res. 3, 379-386 (2010). https://doi.org/10.1007/s12274-010-1042-0

8. Hu, Y., Mao, L., Yuan, X., Lu, J., Chen, R., Chen, T., Zhang, W., Xue, X., Yan, W., Shokouhimehr, M., Zhang, X.L., Jin, Z.: Controllable growth and flexible optoelectronic devices of regularly-assembled $\mathrm{Bi} 2 \mathrm{~S} 3$ semiconductor nanowire bifurcated junctions and crosslinked networks. Nano Res. 13, 2226-2232 (2020). https://doi.org/10.1007/s12274020-2841-6

9. Grigas, J., Talik, E., Lazauskas, V.: X-ray Photoelectron Spectra and Electronic Structure 
of $\mathrm{Bi}_{2} \mathrm{~S}_{3}$ Crystals. Phys. Stat. Sol. 230, 220-230 (2002)

10. Rahman, A.A., Huang, R., Whittaker-Brooks, L.: Distinctive extrinsic atom effects on the structural, optical, and electronic properties of Bi2S3-xSex solid solutions. Chem. Mater. 28, 6544-6552 (2016). https://doi.org/10.1021/acs.chemmater.6b02081

11. Bernechea, M., Cao, Y., Konstantatos, G.: Size and bandgap tunability in Bi2S3 colloidal nanocrystals and its effect in solution processed solar cells. J. Mater. Chem. A. 3, $20642-$ 20648 (2015). https://doi.org/10.1039/c5ta04441c

12. Song, H., Zhan, X., Li, D., Zhou, Y., Yang, B., Zeng, K., Zhong, J., Miao, X., Tang, J.: Rapid thermal evaporation of Bi2S3 layer for thin film photovoltaics. Sol. Energy Mater. Sol. Cells. 146, 1-7 (2016). https://doi.org/10.1016/j.solmat.2015.11.019

13. Whittaker-Brooks, L., Gao, J., Hailey, A.K., Thomas, C.R., Yao, N., Loo, Y.L.: Bi2S3 nanowire networks as electron acceptor layers in solution-processed hybrid solar cells. J. Mater. Chem. C. 3, 2686-2692 (2015). https://doi.org/10.1039/c4tc02534b

14. Liufu, S.C., Chen, L.D., Yao, Q., Wang, C.F.: Assembly of one-dimensional nanorods into Bi2S3 films with enhanced thermoelectric transport properties. Appl. Phys. Lett. 90, 2012-2015 (2007). https://doi.org/10.1063/1.2712504

15. Zhang, B., Ye, X., Hou, W., Zhao, Y., Xie, Y.: Biomolecule-assisted synthesis and electrochemical hydrogen storage of Bi2S3 flowerlike patterns with well-aligned nanorods. J. Phys. Chem. B. 110, 8978-8985 (2006). https://doi.org/10.1021/jp060769j

16. Ellis, L.D., Wilkes, B.N., Hatchard, T.D., Obrovac, M.N.: In Situ XRD Study of Silicon, Lead and Bismuth Negative Electrodes in Nonaqueous Sodium Cells. J. Electrochem. Soc. 161, A416-A421 (2014). https://doi.org/10.1149/2.080403jes

17. Andzane, J., Kunakova, G., Varghese, J., Holmes, J.D., Erts, D.: Photoconductive properties of Bi2S3 nanowires. J. Appl. Phys. 117, (2015). https://doi.org/10.1063/1.4907867

18. Rabin, O., Perez, J.M., Grimm, J., Wojtkiewicz, G., Weissleder, R.: An X-ray computed tomography imaging agent based on long-circulating bismuth sulphide nanoparticles. Nat. Mater. 5, 118-122 (2006). https://doi.org/10.1038/nmat1571 
19. Zhang, L.J., Zhang, B.P., Ge, Z.H., Han, C.G.: Fabrication and properties of Bi2S3-xSex thermoelectric polycrystals. Solid State Commun. 162, 48-52 (2013).

https://doi.org/10.1016/j.ssc.2013.03.013

20. Mizoguchi, H., Hosono, H., Ueda, N., Kawazoe, H.: Preparation and electrical properties of Bi2S3 whiskers. J. Appl. Phys. 78, 1376-1378 (1995).

https://doi.org/10.1063/1.360315

21. Du, X., Cai, F., Wang, X.: Enhanced thermoelectric performance of chloride doped bismuth sulfide prepared by mechanical alloying and spark plasma sintering. J. Alloys Compd. 587, 6-9 (2014). https://doi.org/10.1016/j.jallcom.2013.10.185

22. Kawamoto, Y., Iwasaki, H.: Thermoelectric properties of (Bi1-x Sb x ) 2S3 with orthorhombic structure. J. Electron. Mater. 43, 1475-1479 (2014). https://doi.org/10.1007/s11664-013-2742-5

23. Ge, Z.H., Zhang, B.P., Liu, Y., Li, J.F.: Nanostructured Bi 2-xCu xS 3 bulk materials with enhanced thermoelectric performance. Phys. Chem. Chem. Phys. 14, 4475-4481 (2012). https://doi.org/10.1039/c2cp23955h

24. Yu, Y.Q., Zhang, B.P., Ge, Z.H., Shang, P.P., Chen, Y.X.: Thermoelectric properties of Ag-doped bismuth sulfide polycrystals prepared by mechanical alloying and spark plasma sintering. Mater. Chem. Phys. 131, 216-222 (2011). https://doi.org/10.1016/j.matchemphys.2011.09.010

25. Zhang, Y., Xu, J., Cui, Q., Yang, B.: Eu3+-doped Bi4 Si3 O12 red phosphor for solid state lighting: Microwave synthesis, characterization, photoluminescence properties and thermal quenching mechanisms. Sci. Rep. 7, 1-12 (2017). https://doi.org/10.1038/srep42464

26. Anasane, N., Ameta, R.: Morphologies of nanostructured bismuth sulphide and Mn (II) doped bismuth sulphide nanoparticles: Characterization and application. Mater. Sci. Pol. 35, 6-13 (2017). https://doi.org/10.1515/msp-2017-0032

27. Chen, Y., Wang, D., Zhou, Y., Pang, Q., Shao, J., Wang, G., Wang, J., Zhao, L.D.: Enhancing the thermoelectric performance of Bi2S3: A promising earth-abundant 
thermoelectric material. Front. Phys. 14, (2019). https://doi.org/10.1007/s11467-0180845-4

28. Shubar, M.Y., Saadon, H.L., Abbas, S.J.: High-performance all-optical switching based on nonlinear response in semiconductor Bi2S3-xSex/PMMA nanocomposite films. Mater. Technol. 35, 494-506 (2020). https://doi.org/10.1080/10667857.2019.1701253

29. Song, L., Chen, C., Zhang, S.: Preparation and photocatalytic activity of visible lightsensitive selenium-doped bismuth sulfide. Powder Technol. 207, 170-174 (2011). https://doi.org/10.1016/j.powtec.2010.10.026

30. Chen, X., Hong, Y., Ge, X., Li, C., Miao, X., Wang, P., Zhang, Z., Yin, L.: Se-doped Bi2S3 nanoneedles grown on the three-dimensional carbon foam as a self-supported anode for high-performance sodium ion batteries. J. Alloys Compd. 825, 153901 (2020). https://doi.org/10.1016/j.jallcom.2020.153901

31. Nordström, L., Madsen, G.K.H., Blaha, P., Schwarz, K., Sjöstedt, E.: Efficient linearization of the augmented plane-wave method. Phys. Rev. B - Condens. Matter Mater. Phys. 64, 1-9 (2001). https://doi.org/10.1103/PhysRevB.64.195134

32. Blaha, P., Schwarz, K., Tran, F., Laskowski, R., Madsen, G.K.H., Marks, L.D.: WIEN2k: An APW+lo program for calculating the properties of solids. J. Chem. Phys. 152, 074101 (2020). https://doi.org/10.1063/1.5143061

33. Chu, C.H., Leung, C.W.: The convolution equation of Choquet and Deny on [IN]-groups. Integr. Equations Oper. Theory. 40, 391-402 (2001). https://doi.org/10.1007/BF01198136

34. Perdew, J.P., Burke, K., Ernzerhof, M.: Generalized gradient approximation made simple. Phys. Rev. Lett. 77, 3865-3868 (1996). https://doi.org/10.1103/PhysRevLett.77.3865

35. Bala, A., Nautiyal, T., Auluck, S.: Basic nanosystems of early 4d and 5d transition metals: Electronic properties and the effect of spin-orbit interaction. J. Appl. Phys. 104, 014302 (2008). https://doi.org/10.1063/1.2939251

36. Caracas, R., Gonze, X.: First-principles study of the electronic properties of A2 B3 minerals, with A=Bi,Sb and B=S,Se. Phys. Chem. Miner. 32, 295-300 (2005). https://doi.org/10.1007/s00269-005-0470-y 
37. Li, C., Zhao, J., Hu, Q., Liu, Z., Yu, Z., Yan, H.: Crystal structure and transporting properties of Bi2S3under high pressure: Experimental and theoretical studies. J. Alloys Compd. 688, 329-335 (2016). https://doi.org/10.1016/j.jallcom.2016.06.276

38. Ouerghui, W., Alkhalifah, M.S.: Density functional investigation of structural, electronic, optical and thermodynamic properties of Zn1-xBexO semiconductor. Appl. Phys. A Mater. Sci. Process. 125, 1-12 (2019). https://doi.org/10.1007/s00339-019-2664-z

39. Biswas, K., Zhao, L.D., Kanatzidis, M.G.: Tellurium-free thermoelectric: The anisotropic n -type semiconductor Bi 2S 3. Adv. Energy Mater. 2, 634-638 (2012). https://doi.org/10.1002/aenm.201100775

40. Zhu, H., Pan, M., Johansson, M.B., Johansson, E.M.J.: High Photon-to-Current Conversion in Solar Cells Based on Light-Absorbing Silver Bismuth Iodide. ChemSusChem. 10, 2592-2596 (2017). https://doi.org/10.1002/cssc.201700634

41. Guo, D., Hu, C., Zhang, C.: First-principles study on doping and temperature dependence of thermoelectric property of Bi2S3 thermoelectric material. Mater. Res. Bull. 48, 19841988 (2013). https://doi.org/10.1016/j.materresbull.2013.02.004

42. Cheng, H., Huang, B., Lu, J., Wang, Z., Xu, B., Qin, X., Zhang, X., Dai, Y.: Synergistic effect of crystal and electronic structures on the visible-light-driven photocatalytic performances of Bi2O3 polymorphs. Phys. Chem. Chem. Phys. 12, 15468-15475 (2010). https://doi.org/10.1039/c0cp01189d

43. Ben Abdallah, H., Ouerghui, W.: Hybrid functional calculations of electro-optical properties of novel Ga1-xInxTe ternary chalcogenides. Appl. Phys. A Mater. Sci. Process. 126, 1-12 (2020). https://doi.org/10.1007/s00339-020-03581-8

44. Pejova, B., Grozdanov, I.: Structural and optical properties of chemically deposited thin films of quantum-sized bismuth(III) sulfide. Mater. Chem. Phys. 99, 39-49 (2006). https://doi.org/10.1016/j.matchemphys.2005.10.010

45. Abdallah, H. Ben, Bennaceur, R.: First-principles calculations of the electronic and optical properties of In6S7 compound. Phys. B Condens. Matter. 404, 194-198 (2009). https://doi.org/10.1016/j.physb.2008.10.038 
46. Miller, N.C., Bernechea, M.: Research Update: Bismuth based materials for photovoltaics. APL Mater. 6, 084503 (2018). https://doi.org/10.1063/1.5026541

47. Bushick, K., Mengle, K., Sanders, N., Kioupakis, E.: Band structure and carrier effective masses of boron arsenide: Effects of quasiparticle and spin-orbit coupling corrections. Appl. Phys. Lett. 114, 022101 (2019).

48. Strzałkowski, K., Zakrzewski, J., Maliński, M.: Determination of the exciton binding energy using photothermal and photoluminescence spectroscopy. Int. J. Thermophys. 34, 691-700 (2013). https://doi.org/10.1007/s10765-012-1382-y

49. Sharma, Y., Srivastava, P., Dashora, A., Vadkhiya, L., Bhayani, M.K., Jain, R., Jani, A.R., Ahuja, B.L.: Electronic structure, optical properties and Compton profiles of Bi 2S 3 and Bi 2Se 3. Solid State Sci. 14, 241-249 (2012). https://doi.org/10.1016/j.solidstatesciences.2011.11.025

50. Ouerghui, W., Alkhalifah, M.S., Abdallah, H. Ben: DFT calculations on ZnO1-x compounds for optoelectronic applications. J. Comput. Electron. 20, 467-479 (2021). https://doi.org/10.1007/s10825-020-01645-9

51. Nascimento, V.B., De Carvalho, V.E., Paniago, R., Soares, E.A., Ladeira, L.O., Pfannes, H.D.: XPS and EELS study of the bismuth selenide. J. Electron Spectros. Relat. Phenomena. 104, 99-107 (1999). https://doi.org/10.1016/s0368-2048(99)00012-2

52. Ouerghui, W., Gassoumi, M., Beji, L., Maaref, M.A.: Physica E : Low-dimensional Systems and Nanostructures Optical properties of quaternary GaMnAsP thin layer grown by molecular beam epitaxy. Phys. E Low-dimensional Syst. Nanostructures. 131, 114733 (2021). https://doi.org/10.1016/j.physe.2021.114733

53. Li, C., Shi, G., Song, Y., Zhang, X., Guang, S., Xu, H.: Third-order nonlinear optical properties of Bi2S3 and Sb2S3 nanorods studied by the Z-scan technique. J. Phys. Chem. Solids. 69, 1829-1834 (2008). https://doi.org/10.1016/j.jpcs.2008.01.012 
